\title{
Dynamics of thermal growth of silicon oxide films on $\mathrm{Si}$
}

\author{
R. M. C. de Almeida, S. Gonçalves, and I. J. R. Baumvol \\ Instituto de Física, Universidade Federal do Rio Grande do Sul, Caixa Postal 15051, Porto Alegre, RS, 91501-970, Brazil \\ F. C. Stedile \\ Instituto de Química, Universidade Fedaral do Rio Grande do Sul, Caixa Postal 15051, Porto Alegre, RS, 91501-970, Brazil
}

(Received 14 October 1999)

\begin{abstract}
Thermal growth of silicon oxide films on $\mathrm{Si}$ in dry $\mathrm{O}_{2}$ is modeled as a dynamical system, assuming that it is basically a reaction-diffusion phenomenon. Relevant findings of the last decade are incorporated, as structure and composition of the oxide/Si interface and $\mathrm{O}_{2}$ transport and reaction at initial stages of growth. The present model departs from the well-established Deal and Grove framework [B. E. Deal and A. S. Grove, J. Appl. Phys. 36, 3770 (1965)] indicating that its basic assumptions, steady-state regime, and reaction between $\mathrm{O}_{2}$ and $\mathrm{Si}$ at a sharp oxide/Si interface are only attained asymptotically. Scaling properties of these model equations are explored, and experimental growth kinetics, obtained for a wide range of growth parameters including the small thickness range, are shown to be well described by the model.
\end{abstract}

\section{INTRODUCTION}

Silicon oxide films thermally grown on single-crystalline $\mathrm{Si}(c-\mathrm{Si})$ wafers in dry $\mathrm{O}_{2}$ are still the most common materials used as gate dielectrics in Si-based metal-oxidesemiconductor (MOS) structures. As device dimensions shrink below $0.25 \mu \mathrm{m}$, the oxide films are forced to scale down to thickness of $5 \mathrm{~nm}$ and less. Reliability of highly integrated Si devices is critically dependent on the characteristics of the vitreous oxide film, like thickness uniformity, defect density, dielectric strength, and others, as well as on those of the oxide/Si interface, like roughness, electronic states density, and others. ${ }^{1,2}$ The dielectric performance of ultrathin films of silicon oxide thermally grown on $\mathrm{Si}$ and the structural and electronic properties of the oxide/Si interface have been intensively studied experimentally, theoretically, and computationally ${ }^{3,4}$ in synergism with the development of the semiconductor industry.

Since 1965 the most used theoretical framework to describe silicon oxide thermal growth on $\mathrm{Si}$ in dry $\mathrm{O}_{2}$ has been the Deal and Grove model. ${ }^{5}$ It considers diffusion of an oxidant species $\left(\mathrm{O}_{2}\right)$ through a silicon oxide film from the surface towards the $\mathrm{Si} / \mathrm{SiO}_{2}$ interface. The process is described using constant diffusivity and assuming (i) a steady-state regime, where the gradient of the oxidant species is constant over the oxide film; and (ii) reaction between $\mathrm{O}_{2}$ and $\mathrm{Si}$ at a sharp oxide/Si interface. The result is the well-known linearparabolic law, that agrees with the observed growth kinetics in dry $\mathrm{O}_{2}$ only above a certain oxide film thickness. ${ }^{6-8}$ For thermal oxidation in dry $\mathrm{O}_{2}$, assumption (i) requires an initial oxide layer before the model starts to describe the growth process. This initial oxide layer was estimated between 20 and $30 \mathrm{~nm} .{ }^{5,6}$ The various limitations of this model become important when one departs from thick oxide films, which is the case of films of present and future use in microelectronic devices. To begin with, at initial stages the oxidant species density profiles in the oxide are far from linear, and therefore the steady-state regime cannot be assumed. Furthermore, the interface is not sharp at any stage, ${ }^{9}$ implying that reaction takes place at a finite-width region, known as the reactive layer, ${ }^{10}$ violating another basic assumption of the Deal and Grove model. Also the diffusivity may not remain constant over the whole process, as for example, in thermal growth experiments that take too long in time and are prone to $\mathrm{H}_{2} \mathrm{O}$ contamination. ${ }^{8}$

Description of the growth kinetics in the lower thickness range was addressed by many authors, within the Deal and Grove model framework, adding new terms to the linearparabolic expression in order to fit experimental data. ${ }^{6,7} \mathrm{Al}-$ though fitting the experimental curves, and so providing useful analytical expressions capable of reproducing the whole thickness interval, the extra terms added to the Deal and Grove expression did not have a well-defined physical meaning, even though their dependence on some processing parameters, like temperature, for instance, could be explored. ${ }^{6}$ In order to theoretically explain the early stage kinetics, reaction diffusion equations were explicitly proposed. The solutions were obtained by either assuming a nearly steady state and/or a sharp interface, ${ }^{11,12}$ similarly to the Deal and Grove solution, or else assuming variable diffusivities or reaction rates. ${ }^{13-18}$ Although these models suggest possible physical or chemical phenomena, the simplifying assumptions do not allow an estimate of the relevance of the different processes. None of these models has been shown to be clearly correct and none of them has gained widespread acceptance. One is left then with several expressions that can be used to model the growth kinetics in dry $\mathrm{O}_{2}$ in the thin and ultrathin film regimes well below $20 \mathrm{~nm}$, as well as in further stages of growth, but with no complete dynamical explanation. ${ }^{19,20}$ All those situations not satisfactorily explained in terms of the Deal and Grove model were referred to in the literature as "anomalous," comprising mainly the behavior at initial growth stages.

Recent experimental investigations showed that as film thickness decreases below $20 \mathrm{~nm}$, the contribution to film growth due to reaction away from the interface region becomes increasingly significant. $^{21-26}$ There are strong theoretical and experimental evidences of the existence of a reactive 
layer formed by suboxides (also called Si excess) near the oxide/Si interface. ${ }^{10,27-29}$ Hence, besides steady state, also the sharp interface assumption may be an over simplifying approximation to explain the early stages of oxide growth. The structure of the interface should be obtained as a result rather than given as an assumption of the model. Also, it is desirable to fully understand the exact solution of the model underlying the Deal and Grove framework before other assumptions regarding variable diffusivity and reaction rate are considered.

In the present work finite differences techniques are used to exactly solve reaction-diffusion equations proposed to model the diffusion of an oxidant species through the growing oxide and its reaction to form silicon oxide, as it is described in Secs. II and III. With these equations and their solutions we are able to describe the growth kinetics at all times, from the very beginning to those later stages that were already adequately described by the classical Deal and Grove linear parabolic expression. In the following sections, various aspects of the model are investigated, including scaling properties of the equations, asymptotic behaviors of the solutions, and an operational method is proposed to obtain physical quantities like diffusivity from experimental data. The evolution of the interface width with time can be predicted and the Deal and Grove approximation of sharp interface is shown to be justifiable in the appropriate limits. The idea here is twofold: to provide a theoretical description for the whole thickness interval and a useful tool to make quantitative predictions of film thickness and interface width as functions of pressure, temperature, and time. In Sec. VII, we conclude and discuss possible extensions of the model.

\section{THE MODEL}

The thermal growth of silicon oxide films on $\mathrm{Si}$ is modeled as a dynamical system, assuming that it is basically a reaction-diffusion phenomenon. Since the steady-state regime is not imposed, an initial oxide thickness is not required. Therefore, the model is expected to describe the whole oxide thickness interval. Reaction-diffusion equations have been used to describe different systems, ${ }^{30-32}$ including silicon oxide films thermally grown on $\mathrm{Si}^{1{ }^{1-18}}$ Here they are also applied to the film growth kinetics and exactly solved through numerical iteration. The diffusing species is taken to be $\mathrm{O}_{2}$, to model what has been largely demonstrated by isotopic substitution experiments. ${ }^{21,22,33-35}$ Growth is promoted by reaction of $\mathrm{O}_{2}$ with $\mathrm{Si}$, not necessarily at the oxide/ $\mathrm{Si}$ interface, but wherever the two species meet. The growth kinetics can then be obtained at any temperature by specifying the diffusivity of the $\mathrm{O}_{2}$ molecule in the silica network, $D$, the reaction rate between $\mathrm{O}_{2}$ and $\mathrm{Si}, k$, and the $\mathrm{O}_{2}$ pressure in the gas phase. Growth in one dimension is considered, which is the most common experimental situation, ${ }^{2}$ meaning that as a face of $c-\mathrm{Si}$ is exposed to $\mathrm{O}_{2}$, the silica film grows in the direction perpendicular to this face. The proposed description for the growth kinetics is contained in the following coupled partial differential equations:

$$
\frac{\partial \rho_{O_{2}}}{\partial t}=D \frac{\partial^{2} \rho_{O_{2}}}{\partial x^{2}}-k \rho_{O_{2}} \rho_{S i}, \quad \frac{\partial \rho_{S i}}{\partial t}=-k \rho_{O_{2}} \rho_{S i},
$$

where $\rho_{i}=c_{i} / c_{S i}^{b u l k}$ is the relative concentration of the $i$ species $\left(i=\mathrm{Si}, \mathrm{O}_{2}\right)$ in the solid phase, in units of number of atoms per unit volume, $c_{i}$, normalized by the concentration of $\mathrm{Si}$ in $c-\mathrm{Si}, c_{S i}^{b u l k}$. Thus, the state of any species at any time is characterized by a density function $\rho(x, t)$, where $x$ is the coordinate in the direction of growth, $x=0$ indicating the surface of the sample, and $t$ is the elapsed growth time.

The first equation describes the oxidant species, $\mathrm{O}_{2}$, diffusing through a space initially occupied by crystalline $\mathrm{Si}$, and the subsequent $\mathrm{Si}-\mathrm{O}_{2}$ reaction that takes place whenever the density functions $\rho_{S i}(x, t)$ and $\rho_{O_{2}}(x, t)$ are simultaneously different from zero.

The second equation merely states that silicon is transformed into silica. We do not consider silicon diffusion through the silica film in agreement with recent experimental results, ${ }^{33}$ although short-range (sub-nm) Si interstitial injection from the substrate cannot be excluded. As a consequence of the last assumption we are allowed to write

$$
\rho_{\text {oxide }}(x, t)=1-\rho_{S i}(x, t)
$$

reflecting conservation of $\mathrm{Si}$ species in the $\mathrm{Si}+\mathrm{O}_{2} \rightarrow \mathrm{SiO}_{2}$ reaction. It is assumed that the $\mathrm{O}_{2}$ diffusivity at a certain temperature does not change during oxidation, and therefore $D$ is constant throughout the whole growth process. Another point is that $\rho_{S i}$ may be fractionary, implying the coexistence of silicon and silicon oxide. This is an oversimplified way to account for coexistence of fully and partially oxidized $\mathrm{Si}$, consistently with the existence of suboxides. ${ }^{9,28,29,34,35}$ The interface width is taken as the width of the coexistence region. Furthermore, since oxygen diffuses mainly through oxide, one neglects volume changes due to the above chemical reaction.

Assuming the simple situation of an initially pure silicon surface exposed to a constant gas $\left(\mathrm{O}_{2}\right)$ pressure environment, the initial and boundary conditions then read

$$
\rho_{S i}(x, 0)=1 \quad \forall x \geqslant 0, \quad \rho_{O_{2}}(0, t)=\frac{c_{\text {gas }} f_{v}}{c_{S i}^{\text {bulk }}}=p_{0} \quad \forall t \geqslant 0,
$$

where $c_{\text {gas }}$ is the $\mathrm{O}_{2}$ concentration in the gas phase and $f_{v}$ is the ratio between the accessible free volume for $\mathrm{O}_{2}$ in the silica network and the unit volume of the solid. ${ }^{8}$ Initially there is a pure, $c$-Si substrate from $x=0$ to $\infty$, whose surface is, at all times, exposed to a gaseous medium, $\mathrm{O}_{2}$, with a relative concentration at this surface represented in the second condition by $p_{0}=c_{g a s} f_{v} / c_{S i}^{b u l k}$. As time proceeds, $\mathrm{O}_{2}$ diffuses through the oxide network, reaches $\mathrm{Si}$, reacts producing more oxide, and consequently pushes the oxide/Si interface further in the positive $x$ region. Different values of $D, k$, and $c_{\text {gas }}$ imply different kinetics.

Reaction-diffusion equations to model the thermal oxide growth on Si have already been proposed by other authors. Peng et al. ${ }^{11}$ proposed a model where the interface is taken to be sharp, but whose location is dynamically determined. Using series expansion, Mhetar and Archer ${ }^{12}$ showed that this solution differs from Deal and Grove linear parabolic law by less than $10^{-5}$ percent. On the other hand, Verdi et al. ${ }^{14}$ considered a diffusivity that varies in the near interface region following an ad hoc prescription. The result is in 


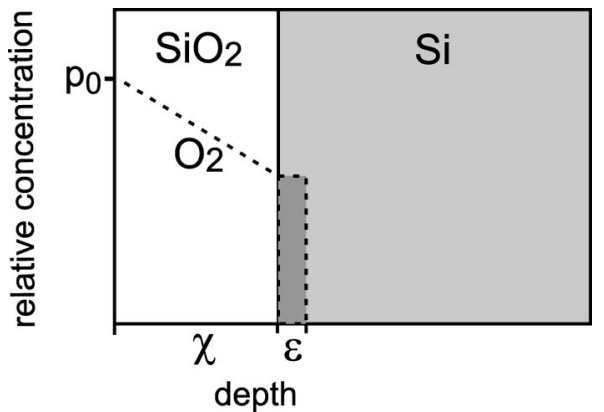

FIG. 1. Oxidant species in the solid phase, silicon oxide (white), and crystalline-Si (gray) profiles assumed to obtain the Deal and Grove limit. Observe that oxidant species and crystalline-Si overlap over a thin region of width $\epsilon$. The form of this region is irrelevant if the area is kept equal to $\left(p_{0}-F \chi\right) \epsilon$.

very good agreement with experimental curves. Dimitrijev and Harrison ${ }^{17}$ considered the emergence of new growth sites at a sharp interface, whose effect is to enhance the reaction rate. Cerofolini et al. ${ }^{18}$ proposed an interface that evolves abruptly to a limit width. This width, however, is not obtained dynamically, but rather it is estimated from experiments. These are valuable interesting suggestions, but the solutions were all obtained by fixing the interface structure as a further assumption. It would certainly be interesting to investigate the exact solution assuming variable diffusivities and/or reaction rates. Nevertheless, here we restrict ourselves to obtain the exact solution with constant $D$ and $k$.

\section{DEAL AND GROVE LIMIT}

We consider a limit situation that yields the Deal and Grove linear-parabolic law, ${ }^{5}$ assuming a linear profile for the oxidant density species and a sharp $\mathrm{SiO}_{2} / \mathrm{Si}$ interface, as shown in Fig. 1. The silicon density profile is taken to be a step function and a small overlap of width $\epsilon$ is assumed between the profiles of silicon matrix and the oxidant species. This overlap is necessary because the $\mathrm{Si}-\mathrm{O}_{2}$ reaction takes place only and whenever the two species meet. In this case, the $\mathrm{O}_{2}$ density function in the solid phase is given by

$$
\rho_{O_{2}}=p_{0}-F x \text { for } x \leqslant \chi(t),
$$

where $\chi(t)$ is the oxide thickness, that is, the depth from the oxide surface where the silicon matrix density profile passes from zero to one, and $F$ is a positive constant over spatial dimension (but depends on time) related to the $\mathrm{O}_{2}$ density gradient in the oxide film. The oxide thickness is given by

$$
\chi(t)=\int_{0}^{\infty} \rho_{\text {oxide }} d x=\int_{0}^{\infty}\left(1-\rho_{S i}\right) d x
$$

and, using Eq. (1), the oxide growth rate can be written as

$$
\frac{d \chi}{d t}=k \int_{0}^{\infty} \rho_{O_{2}} \rho_{S i} d x
$$

Now, considering the situation proposed in Fig. 1, the above integral can be estimated as $\sim \epsilon\left(p_{0}-F \chi\right)$ for small $\epsilon$, that is, the integral in Eq. (6) reduces to

$$
\frac{d \chi}{d t}=\kappa\left(p_{0}-F \chi\right)
$$

where $\kappa=k \epsilon$ is an effective reaction rate with dimensions of length per time, as the usual linear constant of the Deal and Grove model. On the other hand, all oxidant species available from the $\mathrm{O}_{2}$ flux into the overlaping region react with $\mathrm{Si}$ to form oxide. Hence it is also true that

$$
\frac{d \chi}{d t}=-D \frac{\partial \rho_{O_{2}}}{\partial x} \text { for } x=\chi(t)=D F .
$$

Using Eq. (8) in Eq. (7) we get

$$
\frac{d \chi}{d t}=\left(\kappa p_{0}-\frac{\kappa}{D} \frac{d \chi}{d t} \chi\right)
$$

That can be solved and finally yields

$$
\frac{\kappa}{D} \chi^{2}+\chi=\kappa p_{0}\left(t-t_{0}\right)
$$

that has the form of the Deal and Grove linear parabolic law. This solution is valid whenever the approximation implicit in Eq. (7) is valid. Note that the effective reaction rate $\kappa$ should be constant at this asymptotic regime. Hence the Deal and Grove solution is a limit case solution to Eqs. (1), where the chemical species density profiles described in Fig. 1 are assumed. At initial stages of growth, however, this approximation fails, while the reaction-diffusion equations are still valid, and suitable solutions can be obtained numerically, as will be shown in the following. It is interesting to remark that the above solution, Eq. (10), shows a further property of the model: the growth time $t$ can be rescaled by the oxidant species concentration at the oxide film surface, $p_{0}$, as illustrated in what follows.

\section{ADIMENSIONAL EQUATIONS}

Equations (1) form a set of two coupled, nonlinear, second-order, partial differential equations. They are not analytically soluble in general. We have considered an explicit finite difference method and obtained time-dependent solutions. We remark that Eqs. (1) can be written in an adimensional form by considering adimensional variables defined as

$$
\begin{gathered}
\tau=k t, \\
u=\sqrt{\frac{k}{D}} x .
\end{gathered}
$$

Defining $\phi_{i}(u, \tau)=\rho_{i}(x, t)$, the model equations (1) read

$$
\frac{\partial \phi_{O_{2}}}{\partial \tau}=\frac{\partial^{2} \phi_{O_{2}}}{\partial u^{2}}-\phi_{O_{2}} \phi_{S i}, \quad \frac{\partial \phi_{S i}}{\partial \tau}=-\phi_{O_{2}} \phi_{S i}
$$

with boundary and initial conditions given as

$$
\phi_{S i}(u, 0)=1 \quad \forall u \geqslant 0, \quad \phi_{O_{2}}(0, \tau)=\frac{c_{g a s} f_{v}}{c_{S i}^{b u l k}}=p_{0} \quad \forall \tau \geqslant 0,
$$




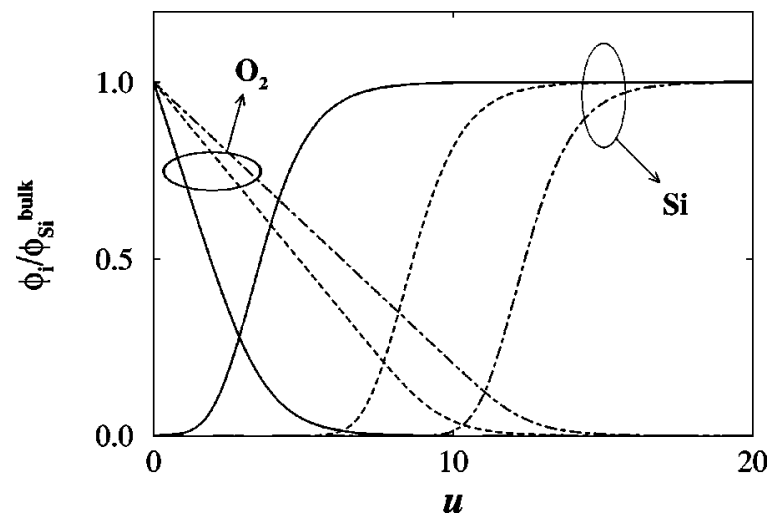

FIG. 2. Calculated $\mathrm{Si}$ and $\mathrm{O}_{2}$ profiles in the solid phase at a given temperature and $\mathrm{O}_{2}$ pressure, for different oxidation times. The solid, dashed, and dot-dashed lines represent, respectively, the profiles at increasing oxidation times. $p_{0}$ was chosen to be equal $\phi_{S i}^{b u l k}$.

and different kinetics are described by a single parameter represented by the relative $\mathrm{O}_{2}$ concentration at the surface $p_{0}$. The adimensional variables are given in terms of "natural" units: $1 / k$ for time and $\sqrt{D / k}$ for length. The adimensional density functions $\phi$ are given in units of the bulk silicon concentration $c_{S i}^{b u l k}$.

\section{NUMERICAL SOLUTION}

In order to obtain concentration profiles and growth kinetics, Eqs. (13) must be solved. The calculation gives then the density profiles $\phi_{i}(u, \tau)$ of all species and the kinetics. Typical profiles at different growth times for a given value of $c_{g a s}$ and temperature are shown in Fig. 2. The $\mathrm{O}_{2}$ profiles are not straight lines as they are assumed to be in the steady-state regime. ${ }^{5}$ Strictly speaking, a steady state is never attained as long as the oxide/Si interface moves deeper into the $c$-Si substrate. Furthermore, this interface is not sharp, in accordance with a wealth of experimental evidences. ${ }^{9,29,34-36}$ In this way, $\mathrm{O}_{2}-\mathrm{Si}$ reaction can take place in the whole region where nonfully oxidized $\mathrm{Si}$ is available. Figure 2 shows that the two hypotheses of the Deal and Grove model are not plausible: (i) steady-state regime is never attained and (ii) a reaction between $\mathrm{O}_{2}$ and $\mathrm{Si}$ leading to growth does not take place at a sharp oxide/Si interface. For high enough temperatures and pressures, and long enough times, the $\mathrm{O}_{2}$ profile approaches a straight line, and the thickness of the interface is much smaller than the oxide thickness. This is the observed situation at oxide thickness well above $20 \mathrm{~nm}$. In this limit, steady-state and abrupt interface are plausible approximations. In other words, asymptotically the Deal and Grove assumptions are valid as it was shown in Sec. III, whereas at initial stages they are not. The kinetics in the initial growth regime, which was described as "anomalous" in the Deal and Grove framework, naturally emerges from the reactiondiffusion equations as follows. Let us consider the quantity that is mainly focused in practical situations: the oxide thickness $\chi$. It can be calculated through the relation below, that is analogous to Eq. (5),

$$
\chi(\tau)=\int_{0}^{\infty} \phi_{\text {oxide }}(u, \tau) d u=\int_{0}^{\infty}\left[1-\phi_{S i}(u, \tau)\right] d u .
$$

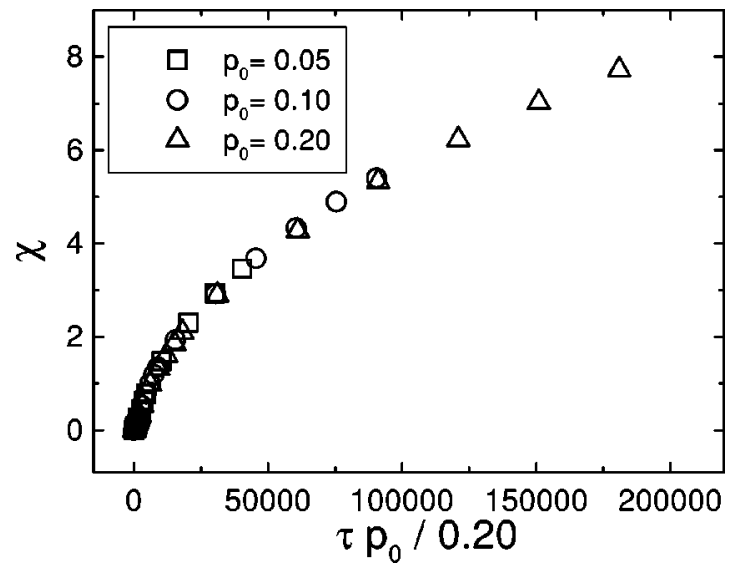

FIG. 3. Kinetic curves obtained theoretically for different values of $p_{0}=0.05,0.10$, and 0.20. All curves were obtained for $D$ $=0.001$ and $k=0.01$.

The oxide growth kinetics $\chi(\tau)$ is completely determined by $\phi_{S i}(u, \tau)$, whose evolution is dictated by Eqs. (13) and therefore is determined by a unique parameter $p_{0}$, the $\mathrm{O}_{2}$ relative concentration at the solid surface.

However, due to the particular form of the differential equations, together with boundary and initial conditions, the system presents a further scaling property. Suppose two different growth kinetics, with $\mathrm{O}_{2}$ concentrations in the solid phase given by $p_{0}^{(1)}$ and $p_{0}^{(2)}=\alpha p_{0}^{(1)}$, then the following relations apply:

$$
\begin{gathered}
\phi_{O_{2}}^{(2)}(u, \tau)=\alpha \phi_{O_{2}}^{(1)}(u, \alpha \tau), \\
\phi_{S i}^{(2)}(u, \tau)=\phi_{S i}^{(1)}(u, \alpha \tau), \\
\chi^{(2)}(\tau)=\chi^{(1)}(\alpha \tau) .
\end{gathered}
$$

Figure 3 shows three different kinetics curves, for different $p_{0}$. This scaling is also in agreement with the asymptotic solution in the Deal and Grove limit given by Eq. (10). Therefore, besides collapsing for different temperatures (i.e., different $D$ and $k$ ) when natural units of the system are used, kinetic curves for different $p_{0}$ collapse to a single curve due to the symmetry present in the form of the reaction-diffusion model equations together with initial and boundary conditions. In summary, the theoretical prediction is that all kinetic curves reduce to a single one.

Initial conditions can be different from one experiment to another due to a native oxide layer of variable thickness present before high-temperature oxidation. We also solved our equations using initial conditions different from Eq. (3), modeling native oxide layers with different thickness and compositions, and different initial interface structures [abrupt or continuously changing from a stoichiometric oxide $\left(\mathrm{SiO}_{2}\right)$ to pure silicon], besides different values for the $\mathrm{O}_{2}$ diffusivity in the native oxide. Although these points are presently under a more exhaustive investigation, the effect of assuming a native oxide layer has been always a shift of the oxide film total thickness by a constant amount. 


\section{COMPARISON WITH EXPERIMENTS}

\section{A. Fitting procedure}

Early stages of growth of silicon oxide films on Si in dry $\mathrm{O}_{2}$ predicted by this model should be tested against experimental data to validate the model. This is accomplished by fitting diverse experimental kinetics with the theoretical curve. For that it is necessary to find the adequate transformation from natural units to usual units, like nanometers and minutes, for each experimental data set, which directly gives the values of diffusivity $D$, reaction rate $k$, and effective $\mathrm{O}_{2}$ concentration $p_{0}$ at the sample surface. Also the native oxide thickness should be estimated. As the samples usually present a relatively thin native oxide film thickness $(\sim 2 \mathrm{~nm}$ or less), whose effect is to shift the growth kinetics curve, the model takes it as a degree of freedom to choose an initial time $\tau_{0}$ and an initial thickness $\chi_{0}$. The choice of $\chi_{0}$ is associated to different native oxide layers that shift the theoretical curve vertically, while the choice of $\tau_{0}$ should be associated with the point on the theoretical curve that represents the instant of the beginning of the oxide film thermal growth process. Actually, these two shifts represent the fitting process, where $D, k$, and $p_{0}$, are scaling factors, being related to the right choice of units.

The fitting for each sample is obtained in the following way:

(i) Numerically iterating Eqs. (13) produces a standard theoretical kinetics curve corresponding to $D=1, k=1$, and $p_{0}=1$.

(ii) The relations between theoretical $\left(\chi_{t h}, \tau_{t h}\right)$ and experimental $\left(\chi_{e x}, t_{e x}\right)$ points are

$$
\chi_{e x}=\chi_{t h} \sqrt{\frac{D}{k}}+\chi_{0}, \quad t_{e x}=\tau_{t h} \frac{1}{k p_{0}}+\tau_{0} .
$$

(iii) Choose two points in the experimental kinetics curve, usually the first and the last.

(iv) Choose a first point in the standard theoretical curve (this is equivalent to choosing $\tau_{0}$ ).

(v) Associating this theoretical point with the first experimental point and using Eqs. (17), determine $k p_{0}$ and a first equation relating $\sqrt{D / k}$ and $\chi_{0}$.

(vi) Using the value of $k p_{0}$ and the second experimental point, determine the second theoretical point and a second equation relating $\sqrt{D / k}$ and $\chi_{0}$.

(vii) Determine $\sqrt{D / k}, \chi_{0}$ and then $\tau_{0}$.

(viii) Rescale the theoretical curve using the values of $\sqrt{D / k}, k p_{0}, \chi_{0}$, and $\tau_{0}$.

(ix) Choose another first point in the standard theoretical curve and repeat steps (v)-(viii) until obtaining the best fit.

Figure 4 shows the result of this fitting procedure for kinetic curves from Ref. 6, corresponding to oxidation in a Joule-effect heated furnace in a variety of growth parameters (temperature and $\mathrm{O}_{2}$ pressure). Since we are concerned with initial stages of growth, we restricted the experimental data shown to the 1-20 nm interval. In Fig. 4(a), data obtained by Kamigaki and Itoh ${ }^{37}$ were also included for $p_{O_{2}}=1 \mathrm{~atm}$. For lower pressures, these kinetics do not coincide with those by Massoud et al. ${ }^{6}$ and we chose the later curves because they have more experimental points. Fits of kinetics obtained by
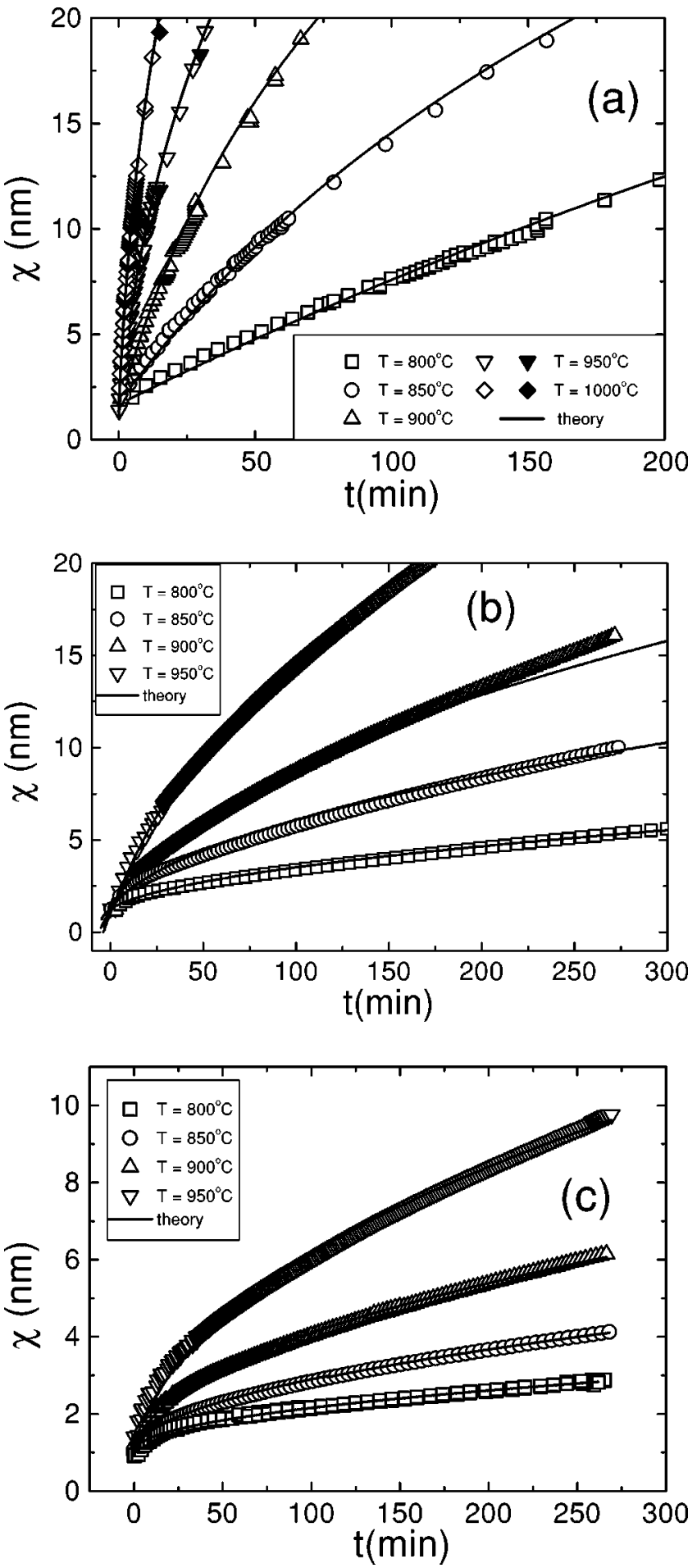

FIG. 4. Theoretical and experimental data comparison for different experiments: open symbols from Ref. 6 and solid symbols from Ref. 37. The theoretical curves were obtained by using the fitting procedure explained in the text. The oxygen partial pressure in the gas phase $p_{O_{2}}$ in experiments was (a) $1 \mathrm{~atm}$, (b) $0.1 \mathrm{~atm}$, and (c) $0.01 \mathrm{~atm}$.

other authors using rapid thermal processing furnace ${ }^{38}$ or Joule heated furnace and a very dry $\mathrm{O}_{2}$ environment ${ }^{23}$ are shown in Fig. 5. Table I lists the extracted values of $D$ and $k$ obtained by the above fitting procedures for the experimental kinetics shown in Figs. 4 and 5, assuming Henry's Law and the free volume hypothesis stated in Eq. (3). 

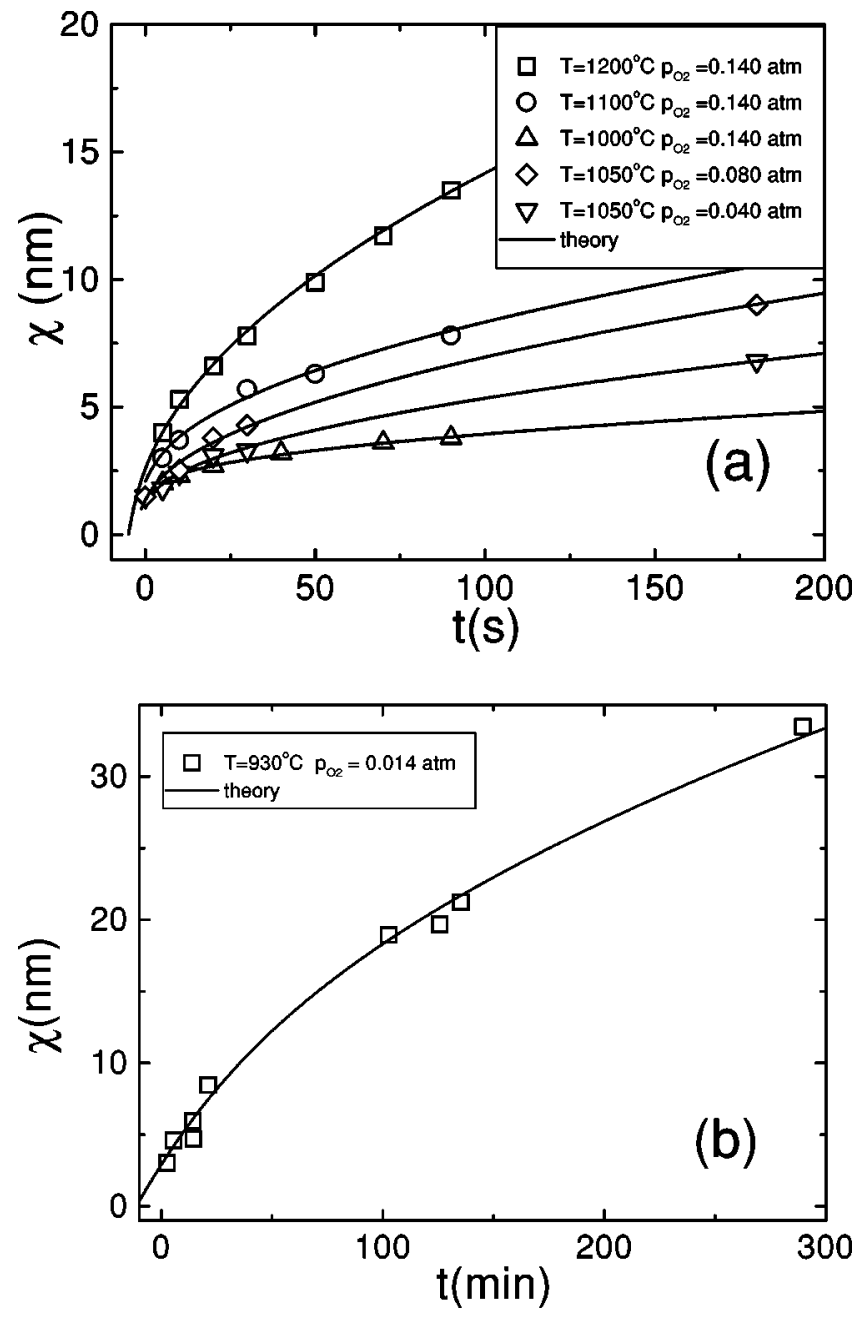

FIG. 5. Growth kinetics by (a) Ganem et al. (Ref. 38) and (b) by Rochet et al. (Ref. 23) (symbols) compared with the theory (solid line).

Figure 6 shows the Arrhenius plot for the diffusivity extracted from the $1 \mathrm{~atm}, 0.1 \mathrm{~atm}$, and 0.01 atm kinetics by Massoud et al. ${ }^{6}$ From the low scattering of the experimental data by Massoud et al. around the linear fit one may conclude that diffusivity is only weakly dependent on pressure. The activation energy extracted from the plot is $W_{0}=46.83$ $\mathrm{kcal} / \mathrm{mol}$, for a Joule heated processing furnace. Different experimental procedures yield different values for the diffusivity and, consequently, may give different values for the activation energy. This should be expected because diffusivity depends on temperature and when a rapid furnace is used the high temperature is attained more rapidly than in the case of a Joule heated furnace.

The kinetics curves are very insensitive to variations in $k$. This is caused by the $k$-dependence of natural units and the scaling properties of the kinetics curve, illustrated in Eqs. (11) and (16). This feature prevents an accurate determination of the reaction constant $k$ and its activation energy.

We emphazise that all the theoretical curves used to fit the experimental curves are the standard curve renormalized to the adequate units and conveniently shifted in time and thickness, to account for the native oxide layer. This means
TABLE I. Oxidation conditions and extracted values of diffusivity coefficient $D$ and reaction rate $k$ extracted from experimental data fitting, as explained in the text.

\begin{tabular}{lccccc}
\hline \hline & $\begin{array}{c}T \\
\text { Data from } \\
\left({ }^{\circ} \mathrm{C}\right)\end{array}$ & $\begin{array}{c}p_{\mathrm{O}_{2}} \\
(\mathrm{~atm})\end{array}$ & $p_{0}$ & $\begin{array}{c}D \\
\left(\mathrm{~nm}^{2} / \mathrm{min}\right)\end{array}$ & $\begin{array}{c}k \\
\left(\mathrm{~min}^{-1}\right)\end{array}$ \\
\hline Fig. 4(a) & 800 & 1 & $3.069 \times 10^{-6}$ & $0.227 \times 10^{6}$ & $3.210 \times 10^{3}$ \\
& 850 & 1 & $2.933 \times 10^{-6}$ & $0.486 \times 10^{6}$ & $1.186 \times 10^{4}$ \\
& 900 & 1 & $2.808 \times 10^{-6}$ & $1.106 \times 10^{6}$ & $3.811 \times 10^{4}$ \\
& 950 & 1 & $2.693 \times 10^{-6}$ & $2.342 \times 10^{6}$ & $1.530 \times 10^{5}$ \\
& 1000 & 1 & $2.587 \times 10^{-6}$ & $5.561 \times 10^{6}$ & $3.131 \times 10^{5}$ \\
\hline Fig. 4(b) & 800 & 0.1 & $3.069 \times 10^{-7}$ & $0.292 \times 10^{6}$ & $9.100 \times 10^{6}$ \\
& 850 & 0.1 & $2.933 \times 10^{-7}$ & $0.568 \times 10^{6}$ & $1.010 \times 10^{7}$ \\
& 900 & 0.1 & $2.808 \times 10^{-7}$ & $1.512 \times 10^{6}$ & $6.310 \times 10^{5}$ \\
& 950 & 0.1 & $2.693 \times 10^{-7}$ & $5.060 \times 10^{6}$ & $3.470 \times 10^{5}$ \\
\hline Fig. 4(c) & 800 & 0.01 & $3.069 \times 10^{-8}$ & $0.193 \times 10^{6}$ & $2.190 \times 10^{7}$ \\
& 850 & 0.01 & $2.933 \times 10^{-8}$ & $0.642 \times 10^{6}$ & $3.130 \times 10^{8}$ \\
& 900 & 0.01 & $2.808 \times 10^{-8}$ & $1.691 \times 10^{6}$ & $1.150 \times 10^{7}$ \\
& 950 & 0.01 & $2.698 \times 10^{-8}$ & $5.150 \times 10^{6}$ & $4.380 \times 10^{6}$ \\
\hline Fig. 5(a) & 1000 & 0.140 & $3.622 \times 10^{-7}$ & $0.378 \times 10^{7}$ & $2.004 \times 10^{8}$ \\
& 1100 & 0.140 & $3.291 \times 10^{-7}$ & $3.768 \times 10^{7}$ & $1.218 \times 10^{8}$ \\
& 1200 & 0.140 & $3.067 \times 10^{-7}$ & $19.56 \times 10^{7}$ & $1.956 \times 10^{8}$ \\
& 1050 & 0.080 & $1.952 \times 10^{-7}$ & $5.643 \times 10^{7}$ & $3.072 \times 10^{8}$ \\
& 1050 & 0.040 & $0.967 \times 10^{-7}$ & $5.535 \times 10^{7}$ & $6.150 \times 10^{8}$ \\
\hline \hline Fig. 5(b) & 930 & 0.014 & $3.656 \times 10^{-8}$ & $5.637 \times 10^{7}$ & $1.656 \times 10^{6}$ \\
\hline \hline
\end{tabular}

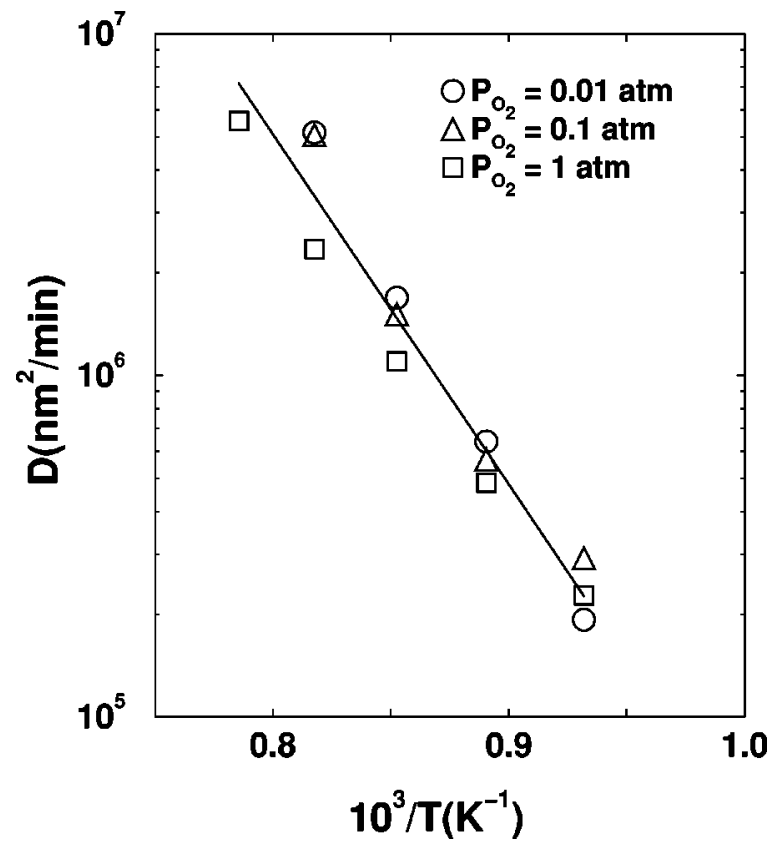

FIG. 6. Arrhenius plot for the diffusivities obtained from the fitting of data by Massoud et al. (Ref. 6) The extracted activation energy is $W_{0}=46.83 \mathrm{kcal} / \mathrm{mol}$ and the preexponential factor $D_{0}$ $=7.96 \times 10^{14} \mathrm{~nm}^{2} / \mathrm{min}$. 
that all these curves can be collapsed into the original standard theoretical curve. A similar collapse was made by Deal and Grove, ${ }^{5}$ assuming initial oxide thickness of $20 \mathrm{~nm}$.

\section{B. Departure from constant diffusivity}

The curves from Ref. 6 contained many more experimental points, extending from 0.1 to $1000 \mathrm{~min}$, and from 1 to 80 $\mathrm{nm}$. For these curves the scaling in pressure predicted by Eqs. (16) is not perfect at long growth times, probably due to an increase in diffusivity. For these experiments, the chosen experimental points for the fitting procedure were not the first and the last ones, but the pair of points that yielded the best fit. This asymptotic departure from the theoretical curve is an expected result, since the present model assumes a constant diffusivity throughout the whole growth process, despite many evidences that this is not the case: (i) transport of the diffusing species through the O-excess region near the surface, ${ }^{22,24,26,39}$ through the Si-excess region near the oxide/Si interface, ${ }^{25,10,34,35}$ and through the stoichiometric $\mathrm{SiO}_{2}$ in the bulk of the growing oxide film should have different diffusivities; (ii) the growth of the defective, nearsurface and near-interface regions saturate within a few $\mathrm{nm},{ }^{22,28,29,36,39}$ while the bulk, stoichiometric oxide grows continuously. So, interstitial diffusion of $\mathrm{O}_{2}$ through the bulk of the growing oxide gives a relative contribution that increases as the width of this region becomes dominant; (iii) oxidation performed in dry $\mathrm{O}_{2}$ flow during long time intervals (many hours) may suffer from water vapor contamination, which accelerates the oxide growth., 83

For long times the solution of Eqs. (1) predicts an inverse growth rate $d t / d \chi=\left(1 / D p_{O}\right) \chi$, with $D$ constant, which is the asymptotic behavior of the Deal and Grove linear parabolic law ${ }^{5}$. In Fig. 7 the inverse growth rate is plotted as a function of oxide film thickness for two different experimental kinetics. ${ }^{6}$ A deviation from a straight line in the thick film range is apparent, corresponding to a variable, increasing diffusivity (as indicated by the dashed lines of different slopes), contrary to the theoretical prediction in this range shown in the inset.

\section{DISCUSSION AND CONCLUSIONS}

From the above results, one can conclude that the thermal growth of silicon oxide films on $c$-Si in dry $\mathrm{O}_{2}$ is promoted by diffusion of $\mathrm{O}_{2}$ through the growing oxide with variable

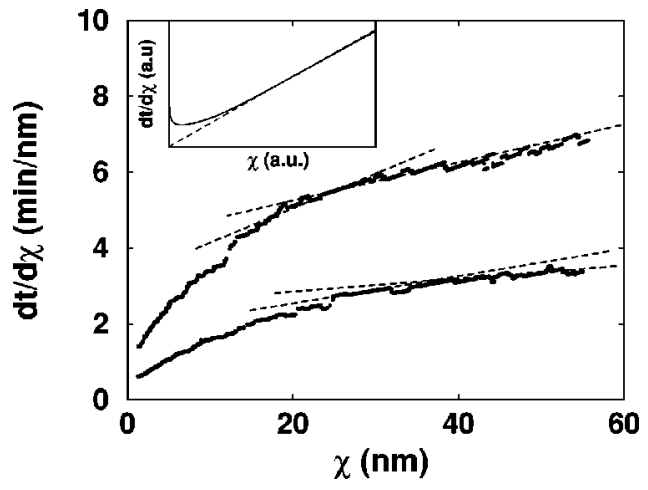

FIG. 7. Experimental inverse growth rates and theoretical inverse growth rate (inset). The experimental data (solid symbols) are taken from Fig. 1 of Ref. 6. The temperatures are $900{ }^{\circ} \mathrm{C}$ and $950{ }^{\circ} \mathrm{C}$ for the upper and lower curves, respectively.

diffusivity. Similar physical arguments could support a variable reaction rate. Nevertheless, even using constant, effective values for the diffusivity and reaction rate, modeling the growth as a reaction-diffusion process where conditions such as initial thickness, steady-state regime, and reaction solely at an abrupt interface are abandoned allows us to reproduce the observed kinetics, especially in the lower thickness (ultrathin film) regime, which is of present and future practical interest.

The present approach opens the possibility of incorporating new and determinant facts on $\mathrm{O}_{2}$ transport at initial stages of thermal oxide growth and on the structure of the oxide/Si interface that were discovered long after the proposal of Deal and Grove ${ }^{5}$ and not yet integrated into growth kinetics models. Some were discussed here, like the graded, $\mathrm{Si}$-excess nature of the oxide/Si interface and the incorporation of freshly arriving $\mathrm{O}_{2}$ into different regions of the growing oxide, while several others may also be included in a reaction-diffusion approach, such as the effect on oxygen transport $^{26}$ caused by oxygen excess centers ${ }^{39}$ in the nearsurface region.

\section{ACKNOWLEDGMENTS}

We thank C. Krug, E. B. O. da Rosa, M. A. Idiart, and L. C. Feldman for fruitful discussions and suggestions. This work has been partially supported by the Brazilian agencies CNPq, FINEP, and FAPERGS.
${ }^{1}$ D. A. Buchanan and S. H. Lo, in The Physics and Chemistry of $\mathrm{SiO}_{2}$ and the $\mathrm{Si}_{-} \mathrm{SiO}_{2}$ Interface-3, edited by H. Z. Massoud, E. H. Poindexter, and C. R. Helms (The Electrochemical Society, Pennington, NJ, 1996), Vol. 96-1, pp. 3-14.

${ }^{2}$ L. C. Feldman, E. P. Gusev, and E. Garfunkel, in Fundamental Aspects of Ultrathin Dielectrics on Si-Based Devices, edited by E. Garfunkel, E. P. Gusev, and A. Vul' (Kluwer, Dordrecht, 1998), pp. 1-24.

${ }^{3}$ K. F. Schuegraf and C. Hu, Semicond. Sci. Technol. 9, 989 (1994).

${ }^{4}$ A. Pasquarello, M.S. Hybertsen, and R. Car, Nature (London) 396, 58 (1998).
${ }^{5}$ B. E. Deal and A. S. Grove, J. Appl. Phys. 36, 3770 (1965).

${ }^{6}$ H. Z. Massoud, J. D. Plummer, and E. A. Irene, J. Electrochem. Soc. 132, 2685 (1985).

${ }^{7}$ C.-J. Han and C. R. Helms, J. Electrochem. Soc. 134, 1297 (1987).

${ }^{8}$ S. Rigo, in Instabilities in Silicon Devices, edited by G. Barbottin and A. Vapaille (Elsevier, Amsterdam, 1986), Vol. 1, pp. 5-100.

${ }^{9}$ M.-T. Tang, K. W. Evans-Lutterodt, M. L. Green, D. Brasen, K. Krisch, L. Manchanda, G.S. Higashi, and T. Boone, Appl. Phys. Lett. 64, 748 (1994). 
${ }^{10}$ A. M. Stoneham, C. R. M. Grovenor, and A. Cerezo, Philos. Mag. B 55, 201 (1986).

${ }^{11}$ K.-Y. Peng, L.-C. Wang, and J.C. Slattery, J. Vac. Sci. Technol. B 14, 3316 (1996).

${ }^{12}$ V. R. Mhetar and L. A. Archer, J. Vac. Sci. Technol. B 16, 2121 (1998).

${ }^{13}$ Y.-L. Chiou, C. H. Sow, and K. Ports, IEEE Electron. Device Lett. 10, 1 (1989).

${ }^{14}$ L. Verdi, A. Miotello, and R. Kelly, Thin Solid Films 241, 383 (1994).

${ }^{15}$ T. K. Whidden, P. Thanikasalam, M. J. Rack, and D. K. Ferry, J. Vac. Sci. Technol. B 13, 1618 (1995).

${ }^{16}$ P. Thanikasalam, T. K. Whidden, and D. K. Ferry, J. Vac. Sci. Technol. B 14, 2840 (1996).

${ }^{17}$ S. Dimitrijev and H. B. Harrison, J. Appl. Phys. 80, 2467 (1996).

${ }^{18}$ G. F. Cerofolini, G. La Bruna, and L. Meda, Mater. Sci. Eng., B 36, 104 (1996).

${ }^{19}$ B. E. Deal, in The Physics and Chemistry of $\mathrm{SiO}_{2}$ and the Si$\mathrm{SiO}_{2}$ Interface, edited by C. R. Helms and B.E. Deal (Plenum, New York, 1988), pp. 5-16.

${ }^{20}$ J. D. Plummer, in The Physics and Chemistry of $\mathrm{SiO}_{2}$ and the Si$\mathrm{SiO}_{2}$ Interface-3, edited by H. Z. Massoud, E. H. Poindexter, and C. R. Helms (The Electrochemical Society, Pennington, NJ, 1996), Vol. 96-1, pp. 129-142.

${ }^{21}$ C.-J. Han and C. R. Helms, J. Electrochem. Soc. 35, 1824 (1988).

${ }^{22}$ I. Trimaille, F. C. Stedile, J.-J. Ganem, I. J. R. Baumvol, and S. Rigo, in The Physics and Chemistry of $\mathrm{SiO}_{2}$ and the $\mathrm{Si}_{-} \mathrm{SiO}_{2}$ Interface-3 (Ref. 20), pp. 59-71.

${ }^{23}$ F. Rochet, S. Rigo, M. Froment, C. D’Anterroches, C. Maillot, H.
Roulet, and G. Dufour, Adv. Phys. 35, 237 (1986).

${ }^{24}$ I. Trimaille and S. Rigo, Appl. Surf. Sci. 39, 65 (1989).

${ }^{25}$ H. C. Lu, T. Gustafsson, E. P. Gusev, and E. Garfunkel, Appl. Phys. Lett. 67, 1742 (1995).

${ }^{26}$ D. R. Hamann, Phys. Rev. Lett. 81, 3447 (1998).

${ }^{27}$ N. F. Mott, S. Rigo, F. Rochet, and A. M. Stoneham, Philos. Mag. B 60, 189 (1988).

${ }^{28}$ F. J. Himpsel, F. R. McFeely, A. Taleb-Ibrahimi, J. A. Yarmoff, and G. Hollinger, Phys. Rev. B 38, 6084 (1988).

${ }^{29}$ T. E. Jackman, J. R. MacDonald, L. C. Feldman, P. J. Silverman, and I. Stensgaard, Surf. Sci. 100, 35 (1980).

${ }^{30}$ D. C. Mattis and M. L. Glasser, Rev. Mod. Phys. 70, 979 (1998).

${ }^{31}$ H. Larralde, M. Araujo, S. Havlin, and H. E. Stanley, Phys. Rev. A 46, R6121 (1992).

${ }^{32}$ G. T. Barkema, M. J. Howard, and J. L. Cardy, Phys. Rev. E 53, R2017 (1996).

${ }^{33}$ I. J. R. Baumvol, C. Krug, F. C. Stedile, F. Gorris, and W. H. Schulte, Phys. Rev. B 60, 1492 (1999).

${ }^{34}$ E. P. Gusev, H. C. Lu, T. Gustafsson, and E. Garfunkel, Phys. Rev. B 52, 1759 (1995).

${ }^{35}$ E. P. Gusev, H. C. Lu, T. Gustafsson, and E. Garfunkel, Appl. Surf. Sci. 104/105, 329 (1996).

${ }^{36}$ F. C. Stedile, I. J. R. Baumvol, I. F. Oppenheim, I. Trimaille, J.-J. Ganem, and S. Rigo, Nucl. Instrum. Methods Phys. Res. B 118, 493 (1996).

${ }^{37}$ Y. Kamigaki and Y. Itoh, J. Appl. Phys. 48, 2891 (1977).

${ }^{38}$ J.-J. Ganem, S. Rigo, and I. Trimaille, Microelectron. Eng. 22, 35 (1993).

${ }^{39}$ A. Stesman and F. Scheerlink, J. Appl. Phys. 75, 1047 (1994). 